\title{
Métodos computacionais baseados em superpixels para segmentação automática da próstata em imagens de ressonância magnética 3D
}

\author{
Giovanni L. F. da Silva ${ }^{1}$, Aristófanes C. Silva ${ }^{1}$, Anselmo C. de Paiva ${ }^{1}$ \\ ${ }^{1}$ Universidade Federal do Maranhão - UFMA, Núcleo de Computação Aplicada - NCA \\ Av. dos Portugueses, SN, Bacanga, 65085-580, São Luís, MA, Brazil \\ \{giovannilucca, ari, paiva\}@nca.ufma.br
}

\begin{abstract}
Prostate segmentation is an essential prerequisite to assist in the diagnosis and treatment of cancer. In current practice, the radiologist performs this process manually, which is extremely time-consuming and requires experience and concentration. Therefore, the development of automatic and reliable methods for prostate segmentation is clinically desirable. Thus, this thesis presents three computational methods for automatic segmentation of the prostate in magnetic resonance images, using image processing and pattern recognition techniques. The proposed methods were evaluated in two public image databases, obtaining a Dice similarity coefficient of $87.67 \%$.
\end{abstract}

Resumo. A segmentação da próstata é um pré-requisito essencial para auxiliar o diagnóstico e o tratamento do câncer. Na prática atual, o radiologista realiza esse processo manualmente, o que é extremamente demorado e exige experiência e concentração. Sendo assim, o desenvolvimento de métodos automáticos e confiáveis para segmentação da próstata é clinicamente desejável. Portanto, esta tese apresenta três métodos computacionais para segmentação automática da próstata em imagens de ressonância magnética, usando técnicas de processamento de imagens e reconhecimento de padrões. Os métodos propostos foram avaliados em duas bases de imagens públicas, obtendo um coeficiente de similaridade Dice de 87,67\%.

\section{Caracterização do problema}

O câncer de próstata é a segunda principal causa de morte por câncer no mundo inteiro entre os homens [INCA 2020]. As estimativas da Sociedade Americana do Câncer para o câncer de próstata nos Estados Unidos, para 2021, são cerca de 248.530 novos casos, apresentando cerca de 34.130 mortes [ACS 2021]. No Brasil, estimam-se 65.840 novos casos de câncer de próstata para cada ano do triênio 2020-2022. Esse valor corresponde a um risco estimado de 62,95 novos casos a cada 100 mil homens [INCA 2020].

Há várias formas de detecção do câncer de próstata, entre elas, as duas maneiras mais comuns para a detecção dessa patologia são o exame do antígeno prostático específico (PSA) no sangue e o exame palpatório. Todavia, esses dois tipos de exames, mesmo realizados em conjunto, não possuem grande precisão, pois dependem que a anatomia da próstata esteja determinada corretamente [Mohler et al. 2016]. Neste contexto, é empregado o uso de exames por imagens da próstata para aumentar a acurácia da detecção, monitoramento e o tratamento do câncer de próstata [Ghose et al. 2012]. 
Entre as modalidades de exames por imagem, a ressonância magnética (RM) é o método mais preciso disponível para obter informações sobre o tamanho, forma e localização da próstata, devido sua capacidade em gerar imagens de alta resolução com excelente contraste de tecidos moles e não envolver radiação ionizante. Na prática atual, a segmentação da próstata na RM é feita principalmente por radiologistas, baseada na inspeção visual fatia por fatia, o que consome tempo, requer um alto grau de habilidade, concentração e é propenso a variabilidade entre radiologistas [Ghose et al. 2012].

A segmentação da próstata é um pré-requisito essencial para auxiliar o diagnóstico e o tratamento do câncer, como a orientação do procedimento de biópsia e a radioterapia. Sendo assim, o desenvolvimento de métodos automáticos e confiáveis é clinicamente desejável [Mohler et al. 2016]. Entretanto, a segmentação automática é desafiadora devido à falta de limites claros da próstata, por causa da aparência semelhante aos tecidos adjacentes e à grande variação no tamanho e na forma entre os diferentes pacientes, por conta das alterações patológicas [Silva et al. 2020].

Por essas razões, nas últimas décadas têm surgido um grande interesse no desenvolvimento e uso de técnicas computacionais que utilizam processamento de imagens e reconhecimento de padrões em imagens de RM, com o objetivo de auxiliar, com aceitável grau de precisão, os resultados de importantes procedimentos médicos. Dessa maneira, a segmentação automática da próstata oferecerá muitos benefícios, como eficiência, agilidade, reprodutibilidade e eliminação de inconsistências entre os radiologistas que realizam a segmentação manual.

\section{Motivação da pesquisa}

No Brasil, o câncer de próstata apresenta a maior incidência entre os homens, sem considerar o câncer de pele não melanoma, e as maiores taxas ocorrem nas regiões nordeste e centro-oeste. A região nordeste apresenta um risco estimado de 72,35 novos casos a cada 100 mil homens, a região centro-oeste possui um risco de 65,29 a cada 100 mil, a região sudeste aponta um risco de 63,94 a cada 100 mil, a região sul exibe um risco de 62 a cada 100 mil, e por fim, a região norte apresenta um risco de 29,39 a cada 100 mil [INCA 2020].

Segundo [Ferlay et al. 2015], a tendência da mortalidade por esse tipo de câncer se apresenta em declínio em quase todas as regiões do mundo, sendo identificada queda de 3,4\%, no período de 2010 a 2014, nos Estados Unidos. No Brasil, ocorreram, em 2017, 15.391 óbitos de câncer de próstata, o equivalente ao risco de 15,25 a cada 100 mil homens. Por se tratar de um tumor com bom prognóstico, a probabilidade de sobrevida em dez anos é encontrada acima de $98 \%$, variando em função de fatores clínicos, genéticos, socioeconômicos e ambientais [INCA 2020].

\section{Objetivos}

O objetivo geral desta tese é desenvolver métodos computacionais para a segmentação automática da próstata em imagens de RM 3D, usando técnicas de processamento de imagens e reconhecimento de padrões.

Destacam-se como objetivos específicos desta tese:

- Detectar a região da próstata para diminuir a região de interesse; 
- Aplicar um algoritmo evolutivo para otimizar a etapa de melhoramento na escolha e ordem das técnicas aplicadas;

- Desenvolver um atlas probabilístico adaptável às diferentes dimensões da RM;

- Segmentar a próstata baseado na classificação dos tecidos presentes na RM.

\section{Contribuições do trabalho}

É de conhecimento geral que quanto antes for detectado e diagnosticado o câncer de próstata, maiores as chances de cura. Por isso, vale ressaltar que o desenvolvimento de sistemas computacionais que auxiliem os radiologistas na segmentação da próstata é de grande importância para a saúde pública nacional. Assim, a implementação dos três métodos propostos nesta tese tem grande relevância para o Brasil uma vez que o mesmo contribui para a divulgação, incentivo e consolidação de uma área de pesquisa científica, a área de informática médica, ainda carente de pesquisadores no país.

No aspecto sócio-econômico, a futura consolidação de um sistema de segmentação automática da próstata utilizando os métodos propostos nesta tese pode contribuir para uma redução significativa dos custos diretos gerados pela hospitalização e intervenções cirúrgicas desnecessárias. Outro benefício a destacar seria a melhoria da qualidade do diagnóstico e tratamento do câncer da próstata baseado em imagens no âmbito médico-hospitalar, contribuindo para diminuir as inconsistências entre os radiologistas que realizam a segmentação manual.

No aspecto científico, a tese apresenta como principais contribuições: 1) otimização da etapa de melhoramento de imagens na escolha e ordem das técnicas aplicadas, 2) desenvolvimento de um atlas probabilístico adaptável às diferentes dimensões da RM, incorporando a informação espacial e anatômica da próstata no processo de segmentação, 3) combinação de técnicas de aprendizado profundo com técnicas convecionais, e 4) descrição da textura dos tecidos de próstata e não próstata usando índices de diversidade.

\section{Resultados e discussão}

Esta tese apresentou três métodos computacionais baseados na classificação dos superpixels [Liu et al. 2018] para a segmentação automática da próstata em imagens de RM. Os resultados obtidos pelos métodos incluem o coeficiente de similaridade Dice (DSC), volume relativo da diferença (RVD), similaridade volumétrica (VS), distância média da superfície (ASD) e a distância de Hausdorff (HD).

O primeiro método proposto apresentou uma abordagem de classificação baseada na técnica de aprendizagem profunda Convolutional Neural Network (CNN) [LeCun et al. 2015] e o algoritmo de otimização por enxame de partículas (PSO) [Eberhart et al. 1995] para otimizar os filtros nas camadas convolucionais. A Tabela 1 apresenta os resultados obtidos na segmentação da próstata com o primeiro método proposto.

O segundo método proposto apresentou uma abordagem de classificação dos superpixels convencional baseada em descritores de textura, usando os índices de diversidade filogenéticos [da Cruz et al. 2020], o algoritmo eXtreme Gradient Boosting (XGBoost) [Chen and Guestrin 2016] e o algoritmo PSO para otimizar os 
Tabela 1. Resultados da segmentação da próstata com o primeiro método proposto.

\begin{tabular}{cccccc}
\hline Exame & DSC $(\%)$ & RVD $(\%)$ & VS & ASD $(\mathbf{m m})$ & HD $(\mathbf{m m})$ \\
\hline Menor & 84,89 & 1,89 & 0,99 & 0,89 & 12,16 \\
Maior & 92,44 & 1,24 & 0,99 & 0,82 & 12,68 \\
Média & $\mathbf{8 7 , 6 7}$ & $\mathbf{2 , 8 3}$ & $\mathbf{0 , 9 6}$ & $\mathbf{0 , 8 9}$ & $\mathbf{1 3 , 6 5}$ \\
Desv. Pad. & $\mathbf{2 , 5 8}$ & $\mathbf{8 , 6 5}$ & $\mathbf{0 , 0 2}$ & $\mathbf{0 , 1 5}$ & $\mathbf{1 , 6 8}$ \\
\hline
\end{tabular}

hiperparâmetros do XGBoost. A Tabela 2 apresenta os resultados obtidos na segmentação da próstata com o segundo método proposto.

Tabela 2. Resultados da segmentação da próstata com o segundo método proposto.

\begin{tabular}{cccccc}
\hline Exame & DSC $(\boldsymbol{\%})$ & RVD $(\boldsymbol{\%})$ & VS & ASD $(\mathbf{m m})$ & HD $(\mathbf{m m})$ \\
\hline Menor & 80,83 & 3,45 & 0,98 & 1,56 & 18,38 \\
Maior & 92,14 & 2,03 & 0,98 & 0,85 & 12,68 \\
Média & $\mathbf{8 5 , 6 4}$ & $\mathbf{7 , 6 8}$ & $\mathbf{0 , 9 6}$ & $\mathbf{1 , 2 2}$ & $\mathbf{1 5 , 1 3}$ \\
Desv. Pad. & $\mathbf{2 , 9 8}$ & $\mathbf{8 , 8 6}$ & $\mathbf{0 , 0 3}$ & $\mathbf{0 , 3 7}$ & $\mathbf{2 , 9 9}$
\end{tabular}

O terceiro método proposto apresentou uma abordagem de classificação dos superpixels híbrida baseada na técnica CNN, o algoritmo XGBoost e o algoritmo PSO para otimizar o tipo de conexão utilizada nas camadas convolucionais. A Tabela 3 apresenta os resultados obtidos na segmentação da próstata com o terceiro método proposto.

Tabela 3. Resultados da segmentação da próstata com o terceiro método proposto.

\begin{tabular}{cccccc}
\hline Exame & DSC (\%) & RVD $(\%)$ & VS & ASD $(\mathbf{m m})$ & HD $(\mathbf{m m})$ \\
\hline Menor & 84,36 & 3,11 & 0,98 & 0,94 & 12,16 \\
Maior & 92,14 & 2,03 & 0,98 & 0,85 & 12,68 \\
Média & $\mathbf{8 7 , 6 5}$ & $\mathbf{3 , 1 8}$ & $\mathbf{0 , 9 6}$ & $\mathbf{0 , 8 8}$ & $\mathbf{1 3 , 5 1}$ \\
Desv. Pad. & $\mathbf{2 , 4 3}$ & $\mathbf{9 , 2 0}$ & $\mathbf{0 , 0 3}$ & $\mathbf{0 , 1 5}$ & $\mathbf{1 , 3 6}$ \\
\hline
\end{tabular}

A Figura 1 apresenta um caso de sucesso na segmentação em todos os métodos propostos. A segmentação do primeiro método está destacada em verde, a segmentação do segundo método está apresentada em amarelo, a segmentação do terceiro método está realçada em azul e a marcação do especialista está delineada em vermelho. Percebe-se que na maioria das fatias intermediárias os métodos obtiveram resultados similares na segmentação, divergindo apenas nas fatias das extremidades do exame, ou seja, região do ápice e região da base da próstata.

Em resumo, o primeiro e o terceiro método apresentaram resultados similares na segmentação da próstata, sendo eles superiores aos resultados obtidos no segundo método. Isso é devido a CNN analisar as características das imagens de forma hierárquica e não se restringir apenas a análise de textura. No entanto, baseado na Tabela 3, o terceiro método 


\section{Figura 1. Exemplo de caso de sucesso na segmentação em todos os métodos propostos.}
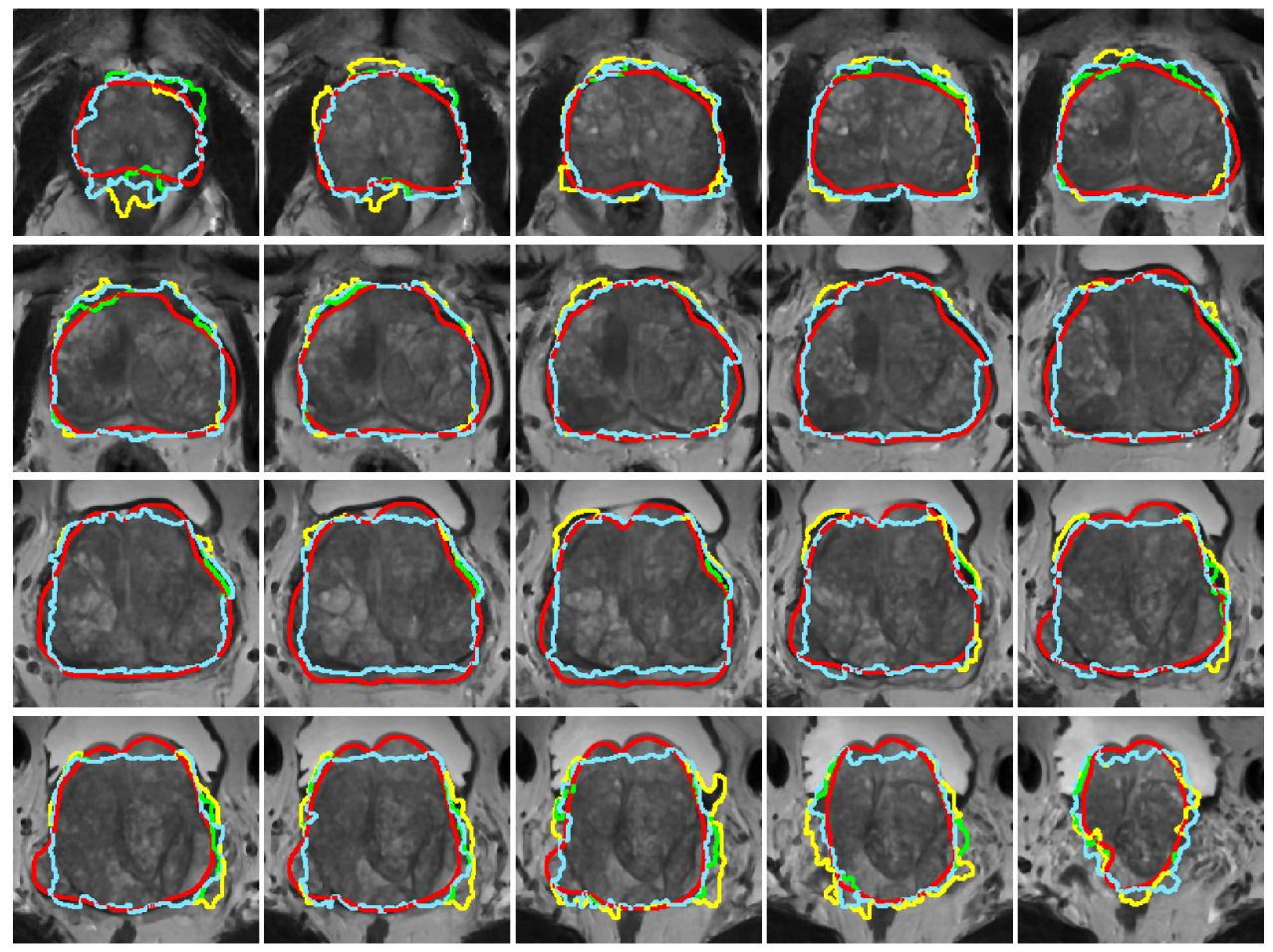

apresenta-se ligeiramente melhor que o primeiro, pois indica um desvio padrão menor nas principais métricas de avaliação DSC e HD, comumente utilizadas na literatura.

Quanto aos resultados obtidos em publicações, a Tabela 4 lista os artigos científicos publicados desde o início do doutorado com relação a segmentação automática da próstata em imagens de RM 3D, com o qualis avaliado na área de engenharias IV. Além desses, existem outros artigos em processo de revisão.

Tabela 4. Artigos publicados em relação a segmentação da próstata.

\begin{tabular}{|c|c|c|c|}
\hline Local & Artigo & Qualis & Status \\
\hline Periódico & $\begin{array}{l}\text { Silva, Giovanni Lucca França da, et al. "Superpixel-based deep convolutional } \\
\text { neural networks and active contour model for automatic prostate segmentation } \\
\text { on 3D MRI scans."Medical and Biological Engineering and Computing. } 2020 .\end{array}$ & A1 & Publicado \\
\hline Congresso & $\begin{array}{l}\text { Silva, Giovanni Lucca França da, et al. "Automatic prostate segmentation on 3D } \\
\text { MRI scans using convolutional neural networks with residual connections and } \\
\text { superpixels.'The 27th International Conference on Systems, Signals and Image } \\
\text { Processing. } 2020 \text {. }\end{array}$ & B1 & Publicado \\
\hline Congresso & $\begin{array}{l}\text { França, João Vitor Ferreira, et al. "Taxonomic indexes for automatic prostate } \\
\text { segmentation on 3D MRI scans using superpixels and probabilistic atlas.'The } \\
\text { 27th International Conference on Systems, Signals and Image Processing. } 2020 .\end{array}$ & B1 & Publicado \\
\hline Congresso & $\begin{array}{l}\text { Ferreira, Jonnison Lima, et al. "Segmentação automática da próstata em } \\
\text { imagens de ressonância magnética utilizando redes neurais convolucionais e } \\
\text { mapa probabilístico." } 18^{\circ} \text { Simpósio Brasileiro de Computação Aplicada à Saúde. } \\
2018 \text {. }\end{array}$ & B4 & Publicado \\
\hline
\end{tabular}




\section{Referências}

ACS (2021). American cancer society: Key statistics for prostate cancer. https://www.cancer.org/cancer/prostate-cancer/about/ key-statistics.html. Online; acessado em 14 de Fevereiro de 2021.

Chen, T. and Guestrin, C. (2016). Xgboost: A scalable tree boosting system. pages 785-794.

da Cruz, L. B., Souza, J. C., de Sousa, J. A., Santos, A. M., de Paiva, A. C., de Almeida, J. D. S., Silva, A. C., Junior, G. B., and Gattass, M. (2020). Interferometer eye image classification for dry eye categorization using phylogenetic diversity indexes for texture analysis. Computer Methods and Programs in Biomedicine, 188:105269.

Eberhart, R. C., Kennedy, J., et al. (1995). A new optimizer using particle swarm theory. In Proceedings of the sixth international symposium on micro machine and human science, volume 1, pages 39-43, New York. New York, NY.

Ferlay, J., Soerjomataram, I., Dikshit, R., Eser, S., Mathers, C., Rebelo, M., Parkin, D. M., Forman, D., and Bray, F. (2015). Cancer incidence and mortality worldwide: sources, methods and major patterns in globocan 2012. International journal of cancer, 136(5):E359-E386.

Ghose, S., Oliver, A., Martí, R., Lladó, X., Vilanova, J. C., Freixenet, J., Mitra, J., Sidibé, D., and Meriaudeau, F. (2012). A survey of prostate segmentation methodologies in ultrasound, magnetic resonance and computed tomography images. Computer methods and programs in biomedicine, 108(1):262-287.

INCA (2020). Tipo de câncer: próstata. http: / /www2 . inca · gov • br/wps / wcm/ connect/tiposdecancer/site/home/prostata. Online; acessado em 27 de Agosto de 2020.

LeCun, Y., Bengio, Y., and Hinton, G. (2015). Deep learning. Nature, 521(7553):436444.

Liu, Y.-J., Yu, M., Li, B.-J., and He, Y. (2018). Intrinsic manifold slic: a simple and efficient method for computing content-sensitive superpixels. IEEE transactions on pattern analysis and machine intelligence, 40(3):653-666.

Mohler, J. L., Armstrong, A. J., Bahnson, R. R., D’Amico, A. V., Davis, B. J., Eastham, J. A., Enke, C. A., Farrington, T. A., Higano, C. S., Horwitz, E. M., et al. (2016). Prostate cancer, version 1.2016. Journal of the National Comprehensive Cancer Network, 14(1):19-30.

Silva, G. L., Diniz, P. S., Ferreira, J. L., França, J. V., Silva, A. C., de Paiva, A. C., and de Cavalcanti, E. A. (2020). Superpixel-based deep convolutional neural networks and active contour model for automatic prostate segmentation on $3 \mathrm{~d}$ mri scans. Medical \& Biological Engineering \& Computing, 58(9):1947-1964. 